Herzschr Elektrophys 2022 - 33:1-2

https://doi.org/10.1007/s00399-022-00842-2 Angenommen: 18. Januar 2022

(C) The Author(s), under exclusive licence to Springer Medizin Verlag GmbH, ein Teil von Springer Nature 2022

\section{Autoren}
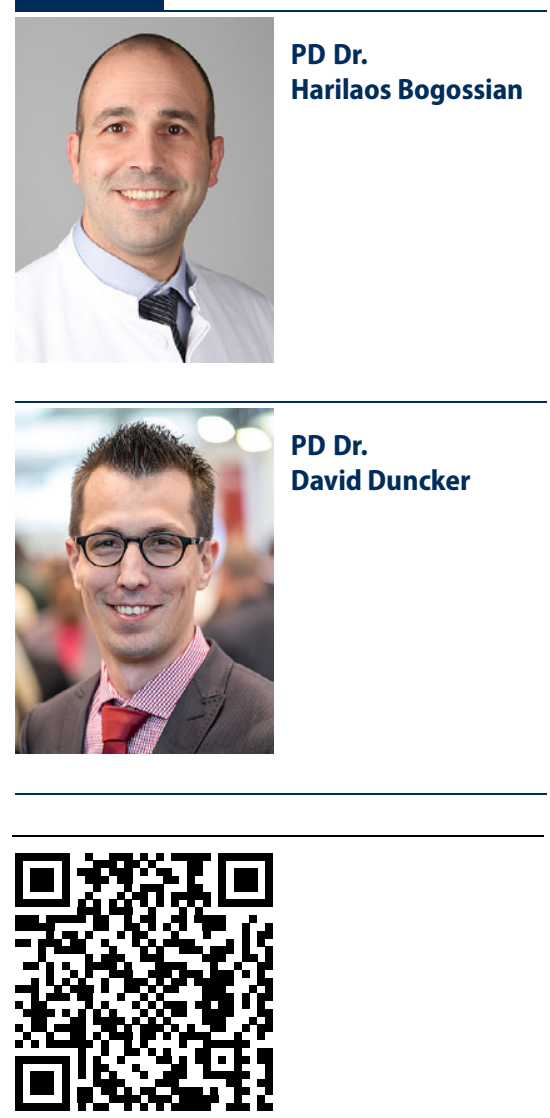

QR-Code scannen \& Beitrag online lesen

\title{
Unaufhaltsame Innovationen in der Elektrophysiologie
}

\author{
Harilaos Bogossian ${ }^{1,2}$. David Duncker ${ }^{3}$ \\ ${ }^{1}$ Klinik für Kardiologie und Rhythmologie, Ev. Krankenhaus Hagen, Hagen, Deutschland \\ ${ }^{2}$ Department of Cardiology, University Witten/Herdecke, Witten, Deutschland \\ ${ }^{3}$ Hannover Herzrhythmus Centrum, Klinik für Kardiologie und Angiologie, Medizinische Hochschule \\ Hannover, Hannover, Deutschland
}

Die Erforschung des Herzens und der Herzkreislauffunktion liegt inzwischen Jahrhunderte zurück. Die interventionelle Diagnostik und Therapie von Herzrhythmusstörungen - im Sinne der Elektrophysiologie - existiert hingegen erst seit einigen Jahrzehnten. Diese Tatsache ist ursächlich für die sehr dynamische Entwicklung im Gebiet der Rhythmologie der letzten Jahre. Die junge Technologie erlebt ihren Aufstieg!

Gegenstand des vorliegenden Schwerpunkts ist es, diese besonderen Fortschritte in der Diagnostik und Therapie von Herzrhythmusstörungen darzustellen. In den letzten zwei Dekaden ist es der Forschung und Entwicklung gelungen, die bildgebenden Verfahren deutlich zu verbessern. Insbesondere die nichtinvasive Bildgebung (mit Magnetresonanztomographie, verbesserten Monitoring-Tools, usw.), aber auch die invasive Diagnostik (mit hochaufIösenden Mapping-Systemen, basierend auf der erhöhten Leistungsfähigkeit der diagnostischen Katheter) haben einen wesentlichen Beitrag zum Verständnis von Herzrhythmusstörungen und den notwendigen Therapieansätze geleistet. Den aktuellen Stand zur MRT-basierten Katheterablation fasst der Beitrag von Khalaph und Kollegen zusammen [1].

Es wurde jedoch im Laufe der Zeit deutlich, dass die Verbesserung der diagnostischen Tools ihre Wertigkeit verliert, wenn nicht potente Therapieoptionen folgen würden. Die Erfolge zu Beginn der Ablationsära waren sehr ernüchternd und die Rezidivraten - insbesondere bei der Behandlung des Vorhofflimmerns und komplexer ventrikulärer Tachykardien enttäuschend.

Viele verschiedene neue Ablationsansätze mit höheren Ablationsenergien, alternativen Energiequellen, aber auch der Zuhilfenahme der Strahlentherapie, waren die Folge.

Die relevanten Entwicklungen der letzten Jahre in diesem Gebiet werden in den Beiträgen von Yahsaly und Kollegen [2], Mathew und Kollegen [3] und Hohmann und Kollegen [4] differenziert vorgestellt. Der neuesten Energieform widmet sich der Artikel von Reinsch und Kollegen: der "pulsed field ablation" [5].

Darüber hinaus erhalten unsere Leserinnen und Leser einen Einblick in die zu erwartende Zukunft einer verbesserten diagnostischen Datenverarbeitung. Die Möglichkeit, immer größere Datenmengen mit immer komplexeren Computeranalysen zu verarbeiten und zu verknüpfen, ist Gegenstand zahlreicher Forschungsprojekte. Klinisch tätige Ärztinnen und Ärzten sollen in Zukunft damit bei der Diagnosestellung im Alltag unterstützt werden.

Im vorliegenden Schwerpunkt dieser Ausgabe der Herzschrittmachertherapie +Elektrophysiologie wird explizit auch auf die Weiterentwicklung in diesen Bereichen eingegangen: In diesem Zusammenhang werden die Themen „Big Data, Machine Learning und künstliche Intelligenz in der Elektrophysiologie" von Isaksen und Kollegen [6] sowie Nedios und Kollegen [7] präsentiert und diskutiert.

Unter Berücksichtigung dieser Fortschritte im Bereich der Elektrophysiologie ist es legitim, über die unaufhaltsamen 


\section{Editorial}

Innovationen unseres Arbeitsfeldes zu sprechen.

Wir wünschen Ihnen eine spannende Lektüre.

Ihre

Harilaos Bogossian und David Duncker

\section{Korrespondenzadresse}

\section{PD Dr. Harilaos Bogossian}

Klinik für Kardiologie und Rhythmologie, Ev. Krankenhaus Hagen

Brusebrinkstr. 20, 58135 Hagen, Deutschland bogossian@evk-haspe.de

\section{PD Dr. David Duncker}

Hannover Herzrhythmus Centrum, Klinik für Kardiologie und Angiologie, Medizinische Hochschule Hannover

Carl-Neuberg-Str. 1, 30625 Hannover, Deutschland

duncker.david@mh-hannover.de

Interessenkonflikt. H. Bogossian und D. Duncker geben an, dass kein Interessenkonflikt besteht.

\section{Literatur}

1. Khalaph M, Guckel D, Bergau L et al (2022) MRTbasierte Katheterablation. Herzschr Elektrophys. https://doi.org/10.1007/s00399-021-00832-w

2. Yahsaly L, Siebermair J, Wakili R (2022) Die Katheterablation: Entwicklung und Auswahl verschiedenerVerfahren (HPSD, extrakalt, bipolar). Herzschr Elektrophys. https://doi.org/10.1007/ s00399-022-00843-1

3. Mathew S, Müller P, Hardy C et al (2022) Update Ablation ventrikulärerTachyarrhythmien. Herzschr Elektrophys. https://doi.org/10.1007/s00399022-00840-4

4. Hohmann S, Hillmann HAK, Müller-Leisse J et al (2021) Stereotactic radioablation for ventricular tachycardia. Herzschr Elektrophys. https://doi.org/ 10.1007/s00399-021-00830-y

5. Reinsch N, Füting A, Höwel D et al (2022) Pulsed field ablation. Herzschr Elektrophys. https://doi. org/10.1007/s00399-021-00833-9

6. Isaksen JL, BaumertM, Hermans ANLetal (2022) Artificial intelligence in electrophysiology — current knowledge and perspectives. Herzschr Elektrophys. https://doi.org/10.1007/s00399-02200839-x

7. Nedios S, lliodromitis K, Kowaleski Cetal (2022) Big data in electrophysiology. Herzschr Elektrophys. https://doi.org/10.1007/s00399-022-00837-z

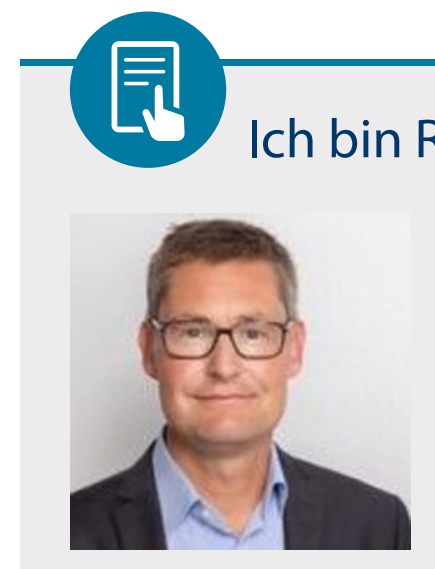

„... es einer der spannendsten Bereiche der Kardiologie ist. Es ist mit einer Reise um die Erde vergleichbar. Wer zu dieser Reise einmal angetreten ist, findet kein Ende. Wer nicht täglich etwas Neues sieht oder lernt, der macht etwas falsch. Man trifft auf sehr junge wie ältere Menschen, denen man helfen kann und die teilweise sogar geheilt werden. Alles zusammen: ein nicht endendes Glück für einen Arzt. Nehmen Sie möglichst Viele mit auf diese faszinierende Reise."

Prof. Dr. med. Lars Eckardt, Klinik für Kardiologie II - Rhythmologie, Universitätsklinikum Münster 